\title{
Historia
}

\section{La formación de médicos y cirujanos durante los siglos XVI a XIX: Las Escuelas Prácticas de Medicina y Cirugía en el Perú}

\author{
Miguel Rabí ${ }^{1}$
}

Resumen

Palabras clave

\begin{abstract}
Se hace un completo estudio sobre las Escuelas Prácticas de Medicina y Cirugía que se desarrollaron en Lima y en algunas principales ciudades del Virreinato, su evolución histórica y social desde el S. XVI hasta el S. XVIII, en la etapa anterior al establecimiento del Colegio de Medicina y Cirugía de San Fernando (1808). Se menciona los principales médicos y cirujanos americanos egresados de estas Escuelas y el valioso aporte al conocimiento de la ciencia y la investigación que ellas realizaron.
\end{abstract}

Escuelas médicas; Perú, escuelas médicas; educación médica; Perú, historia de la medicina; cirugía/historia.
Physicians and surgeons formation from the XVI through XIX centuries: The Medicine and Surgery Practical Schools in Peru

\section{Abstract}

This document presents a complete study on the Practical Schools of Medicine and Surgery in Lima and some other important Vice Royalty cities. It covers the historical and social evolution from the XVI through the XVIII centuries, and the period before the establishmentoftheSanFernando College ofMedicine andSurgery in 1808. The most important American creole doctors and surgeons graduating from these Practical Schools are mentioned as well as their contribution to medical science and research in public health is also described.

Key words: Schools, medical; Perú, schools, medical; education, medical; Peru, history of medicine; surgery/history.

\footnotetext{
Profesional sanmarquino, con estudios de posgrado en España; investigador de la Historia de la Medicina Peruana; funcionario (r) del Ministerio de Salud y de Organismos Internacionales; miembro de la Asociación de Historia de la Medicina Peruana y de Instituciones culturales, académicas y profesionales de Perú y España; autor de la serie Historia de la Medicina Peruana, con ocho tomos publicados y cuatro en proceso de edición. Universidad Nacional Mayor de San Marcos, Facultad de Medicina. Lima, Perú.
}

La Historia de la Medicina Peruana contiene un enorme conjunto de acontecimientos, muchos aún desconocidos, vinculados con la protección y cuidado de la salud de las personas. Al profundizar las investigaciones sobre los siglos XVI a XIX, descubrimos nuevas facetas, con contenidos no bien interpretados y aún distorsionados. No obstante las dificultades confrontadas por la dispersión y carencia documental, encontramos importantes datos sobre la formación, cultura y desarrollo occidental que España trasladó tempranamente al Nuevo Mundo.

Paralelamente, encontramos los acontecimientos generados en el ámbito americano; unos como consecuencia del mestizaje integrador; y otros, que debieron adoptarse en función de las circunstancias y de las exigencias del medio social, cultural, político y económico, por separado del régimen tutorial clásico. De éstos hemos seleccionado para el presente estudio el de la formación de médicos y cirujanos criollos, mestizos y de otras castas; y comentamos el proceso de compensación de la carencia 
de un centro de preparación profesional (Colegio de Medicina y Cirugía), en la relación médico-estudiante. Nos referimos a las Escuelas Prácticas de Medicina y Cirugía en el periodo comprendido desde 1551 hasta 1808 , por consiguiente anterior al establecimiento del Colegio de Medicina y Cirugía de San Fernando en la Ciudad de Lima a partir de 1808*.

Entre las Cátedras que se leía en la Real Universidad de San Marcos de Lima, desde su establecimiento formal, en 1551 , se dio especial importancia a las de Medicina (Prima y Vísperas); para la formación de profesionales en América Meridional, cuya necesidad siempre fue evidente. Entre los años 1571 y 1808, un importante conjunto de profesionales (médicos y cirujanos) realizó estudios en la Universidad, reconocidos previo examen por el Protomedicato y autorizados para ejercer en todo el Perú. La interrogante que se formula es conocer cómo se cumplieron las prácticas hospitalarias y asistenciales exigidas durante tres años como mínimo, cómo se otorgó las certificaciones de estudio, aprendizaje y destreza profesional, quién las concedía, qué trámites y condicionamientos existían, etc. Preguntas que trataremos de esclarecer, merced al examen exhaustivo de las Constituciones de la Universidad, de los Hospitales, así como de los expedientes de visitas de control y fiscalización de cuentas realizadas ${ }^{1}$ ).

\section{Los hospitales mayores y menores}

El establecimiento de la red de Hospitales Mayores y Menores se inició en el Virreinato del Perú, desde 1533 en adelante, para atender las necesidades sanitarias de la población, hacer frente a las pestes y epidemias frecuentes, proporcionar el tratamiento más adecuado de la medicina occidental y aplicar los medicamentos necesarios para recuperar la salud, dando espe- cial atención a los naturales o indígenas (hombres, mujeres, niños), tanto por derecho propio como por constituir responsabilidad y obligación de la Autoridad gubernativa. La normatividad en este campo fue abundantísima, desde que se exigió el compromiso obligatorio de todas los funcionarios de velar por el cuidado, conservación, recuperación y mejora de la salud de la población indígena, además de su adoctrinamiento y enseñanza moral cristiana, extirpación de las herejías e idolatrías y su preparación para la nueva estructura social, política y económica establecida $\left({ }^{2}\right)$.

El desarrollo de los servicios asistenciales fue temprano, paralelo con el establecimiento de las nuevas poblaciones; fue la respuesta inmediata a las necesidades de la población, sobre todo para hacer frente a las numerosas enfermedades que periódicamente afectaban la costa y sierra del Perú. Los gérmenes patógenos procedentes del Viejo Mundo fueron transmitidos en el intenso tráfico de personas, animales, bienes, mercancías, naves, etc, generando grandes problemas sanitarios en un medio nuevo carente de resistencia: viruela, gripe o influenza, sarampión, garrotillo, fiebres recurrentes, tifus o tabardillo, sífilis, además de las propias del medio, como tuberculosis, malaria o paludismo, disenterías o cámaras, uta o espundia, venéreas o trepone-matosis, verrugas, etc., que ocasionaron elevada mortandad y por consiguiente el descenso poblacional $\left({ }^{3,4}\right)$.

Sin dejar de considerar las enfermerías de campaña o de sangre establecidas entre 1530 y 1535 y en otras acciones, la extensa red de servicios hospitalarios destinada a la población se inició en 1533, como se aprecia en el siguiente resumen:

1533 Casa Enfermería en Tumbes.

1534 Enfermería provisional en Jauja.

1538 Casa Enfermería, primer Hospital en Lima.

1546 Hospital de la Caridad de naturales, Cuzco.

* Existe numerosa bibliografía nacional e internacional sobre el tema. En el presente estudio, se menciona la más importante y significativa. Para mayores referencias consultar las referencias bibliográficas consignadas en los ocho tomos de nuestra serie Historia de la Medicina Peruana, que venimos publicando desde 1997. 
1549 Hospital de Santa Ana de los naturales (hombres y mujeres), Lima.

1550 Hospital de San Andrés (hombres) Lima.

1551 Hospital de San Sebastián, en Trujillo.

1553 Recogimiento de San Juan de la Penitencia, para Doncellas en Lima.

1555 Hospital de San Lázaro, en Cuzco.

1555 Hospital del Espíritu Santo en el Cuzco, para los naturales.

1555 Hospital de Huamachuco para los naturales, en Trujillo.

1556 Hospital de naturales, Huamanga, Ayacucho.

1556 Hospital de Santa Ana y de San Nicolás en Ica, para naturales.

1557 Hospital de Na. Sa. de los Remedios, Cuzco.

1559 Hospital de Santa María de la Caridad, Lima (mujeres).

1563 Hospital de San Lázaro, Lima (leprosos).

1570 Hospital de San Sebastián para naturales, en Zaña.

1575 Hospital del Espíritu Santo, Lima (gente de mar y su familia).

1578 Hospital de Santa Ana en Chachapoyas, para naturales.

1580 Hospital de San Nicolás en el Puerto del Callao, para naturales.

1580 Hospital de San Nicolás en Chancay, para naturales.

1585 Hospital de Cailloma en Arequipa, para naturales.

1593 Hospital de Convalecencia de San Diego, en Lima (hombres).

1594 Hospital de San Pedro para sacerdotes, en Lima.

1594 Hospital de Castrovirreina, en Huancavelica, para naturales,

1595 Hospital de San Bartolomé para naturales, en Huancavelica.

1598 Hospital de Niños Huérfanos y Expósitos, en Lima.

1600 Hospital de naturales, en Cañete.

1600 Hospital de naturales, en Huánuco.

1602 Hospital de San Juan de Dios, en Arequipa (hombres y mujeres).

1613 Hospital de San Antonio, en Pisco (hombres y mujeres).

1615 Hospital de San Antonio, en Arica (hombres y mujeres).

1617 Hospital de San Bartolomé para hombres, en el Cuzco.

1620 Hospital Na. Sa. de la Piedad para naturales, en Cajamarca.

1629 Hospital de San Andrés para mujeres, en el Cuzco.

1646 Hospital de San Bartolomé en Lima para la gente de color (hombres y mujeres).
1648 Hospital de Convalecencia Na. Sa del Carmen para naturales, Lima.

1650 Hospitales de San Agustín y San Francisco, en Huamanga.

1659 Hospicio de Niñas Santa Cruz de Atocha, en Lima (huérfanas).

1669 Hospital Refugio de Incurables, en Lima (hombres y mujeres).

1670 Recogimiento de Amparadas para mujeres, en Lima.

1676 Hospital de Na. Sa. de Belén, en Chachapoyas, para naturales.

1677 Hospital de Cajamarca para mujeres.

1678 Hospital de Huanta, Ayacucho, para naturales.

1687 Hospital de La Almudena, en el Cuzco.

1687 Hospital de Huaraz para los naturales.

1690 Hospital de Asillo, Collao, Puno, para naturales.

1695 Hospital de Moyobamba para naturales.

1700 Hospital de Lambayeque para naturales.

1710 Hospital de los Camilos o de la Buena Muerte o de Agonizantes en Lima.

1759 Hospicio para pobres hombres y mujeres, en Lima.

1770 Hospital de Bellavista en el Callao para toda la población.

1780 Hospital de Camilas o Buena Muerte, en Lima, para mujeres.

1785 Hospicio y Colegio para doncellas en Huamachuco, Trujillo.

1787 Hospital de mujeres y niñas en la ciudad de Ica.

1788 Hospital Santa María del Socorro, en Ica, para mujeres.

1792 Hospital de Sicuani, Cuzco, para hombres y mujeres.

1792 Anfiteatro Anatómico, Hospital de San Andrés, Lima.

1808 Nuevo Colegio de Medicina y Cirugía de San Fernando, Lima $\left({ }^{5}\right)$.

Los primeros médicos y cirujanos que prestaron servicios y atendieron a la población tanto natural o autóctona como a la recién establecida, fueron profesionales españoles premunidos de los correspondientes títulos y autorizaciones de ejercicio, que trasladaron el vasto conocimiento de la medicina occidental; ellos fueron portadores de invalorables textos y recopilaciones sobre el arte curativo, así como de los medicamentos simples y compuestos requeridos para las circunstancias; a los que se fueron agregando los oriundos del Nuevo Mundo, cuyo primer estudioso y analista 
fue el célebre Nicolás Monardes, en Sevilla, con la colaboración de Pedro de Osma Xara y Cejo, desde el Perú $\left({ }^{6}\right)$.

Citemos algunos nombres de los primeros médicos que llegaron al Perú: Hernando de Sepúlveda (Protomédico sustituto), Jerónimo Enríquez, Bachiller Marín, Francisco Franco y Pedroza, Pedro Gago de Vadillo, Francisco de Alva, Álvaro de Torres, Gaspar de Meneses, Antonio Sánchez de Renedo (Protomédico titular), Jerónimo de Aguilar, Francisco López de Nájera, Juan de Álvarez, Francisco Gutiérrez, Alonso Gutiérrez, Hernando de Aguilar, Francisco de San Juan, Antonio de Albizu y muchos más, durante el Siglo XVI $\left({ }^{7}\right)$.

Junto con ellos, aparecieron también los boticarios, barberos, flebótomos, albéitares, ensalmadores, prácticos, etc, que intervenían en una y otra forma en los procesos curativos; por lo que, el Cabildo de la Ciudad de Lima debió dictar medidas de control, la presentación de sus títulos, examen de conocimientos, etc, concertando con el Protomédico la revisión exhaustiva y el examen previo, antes de conceder el permiso de ejercicio o de libre actividad. Igual medida se adoptó con las boticas, al disponerse la revisión del buen estado, calidad y precio justo de los principios activos, simples y compuestos, empleados en la elaboración de las fórmulas magistrales. En cambio, siguiendo las orientaciones trazadas por el benemérito Fray Jerónimo de Loayza, quien estableció la primera botica en el Hospital de Santa Ana de los naturales, los demás establecimientos contaron con centro propio para la elaboración de los medicamentos; el frecuente tráfico de naves facilitó la adquisición de los insumos requeridos, junto con los libros más destacados de la farmacopea universal (Modus Faciendi, Mesué, Galeno, Avicena, Ávila, Palacios, etc) $\left({ }^{8}\right)$.

Observamos así que, desde el primer periodo de contacto y luego de asentamiento, se había iniciado el proceso de intercambio científico, merced a la observación, estudio y experimentación de casos, modalidades de tratamiento y comparación con los métodos utilizados por los naturales; labor que realizaron muchas personas, como Pedro de Osma Xara y Cejo, con Nicolás Monardes, Pedro Cieza de León, Garcilaso Inca de la Vega, Montesinos, Bernabé Cobo, José de Acosta, Antonio Vázquez de Espinosa, etc. Al establecerse, en 1570, formalmente, el Protomedicato, con su titular Antonio Sánchez de Renedo, asumió el control de los títulos profesionales y el reconocimiento de profesionales, visitas a las boticas y control de los precios, junto con la investigación y estudio de los sistemas curativos de los naturales. El mundo occidental llegó así a conocer y utilizar progresivamente el inmenso aporte realizado por el Nuevo Mundo en alimentos y en medicamentos $\left({ }^{1}\right)$.

La Universidad y Estudio General de Lima, que venía cumpliendo desde 1548 acción formativa y orientadora de la juventud, al amparo de la Religión Dominica, tanto en Cuzco como en Lima, tuvo feliz aprobación por la Real Cédula de 12 de mayo de 1551, del Emperador Carlos, merced a las gestiones del Cabildo encomendadas a Fray Tomás de San Martín y al Cap. Jerónimo de Aliaga, en beneficio de los hijos nacidos en las nuevas tierras, evitando el largo, costoso y arriesgado viaje a España. Comenzaba en esta forma el proceso de incorporación a la Universidad de los jóvenes criollos, mestizos, incluyendo los naturales, por estar plenamente equiparados y reconocidos sus legítimos derechos en todo el extenso Virreinato $\left({ }^{2}\right)$.

Se establecieron los hospitales y las escuelas de primeras letras junto con los Colegios (bajo el manto protector de las Religiones), a seguido y simultáneamente con la Universidad. Mas, anotemos que el gran cambio y secularización de la Universidad se produjo entre los años 1569 y 1571, cuando integraban el Claustro tres importantes médicos residentes en Lima (Francisco Franco, Gaspar de Meneses y Antonio Sánchez de Renedo), autores de las primeras Constituciones en 1571, y procedieron a la elección del primer Rector seglar, don Pedro Fernández de Valenzuela ${ }^{(1)}$. 
Con fundamento, los historiadores han expresado que los procesos de transformación y renovación intelectual, política y cultural en el Perú, se generaron entre los profesionales de la salud y del derecho: representados por el Colegio de Medicina y Cirugía de San Fernando y por el Convictorio de San Carlos, pilares fundamentales de la Universidad de San Marcos de Lima $\left({ }^{9}\right)$.

\section{Visión del siglo XVI}

En el curso de los primeros 65 años de existencia de la Ciudad de Lima, su expansión fue considerable, pues de los 14 vecinos iniciales, en 1535 , a fin de siglo contaba con 14262 habitantes, 314 hectáreas de extensión, 168 calles, 117 manzanas, 6 pilas grandes públicas, 17 pilones públicos corrientes, 6 Conventos (Santo Domingo, La Merced, San Agustín, la Compañía de Jesús, San Francisco y Montserrat), 3 Recolecciones (Descalzos, Belén y Recoleta Dominica), 5 Monasterios (Encarnación, Concepción, Trinidad, Descalzas y Santa Clara), 6 Colegios Mayores (San Felipe, San Martín, Santo Toribio, San Ildefonso, San Pablo, San Buenaventura), 2 ermitas (Del Prado y Guadalupe), 28 Iglesias, grandes edificios públicos (Palacio del Virrey y Real Audiencia, Casa del Cabildo, la Universidad, Casa del Arzobispo, Tribunal de la Inquisición, cárceles públicas y de Corte, rastros o mataderos, corral de comedias, Casa del Consulado, alhóndiga, nieve, etc.) y 8 Hospitales (Santa Ana, San Andrés, La Caridad, Espíritu Santo, San Lázaro, Huérfanos, San Diego o San Juan de Dios, San Pedro de Sacerdotes). Asimismo, contaba con Colegios para doncellas, recogimientos para mujeres abandonadas y divorciadas, Colegios menores o de primeros estudios, en torno a los Conventos, albergues, posadas, centros de hospedaje, etc. $\left({ }^{10}\right)$.

\section{Hospitales mayores}

Era la denominación propia de los grandes establecimientos de salud, donde se atendía el común de las personas, por do- lencias, padecimientos y accidentes; contaban con los servicios básicos indispensables en sus enfermerías o salas, para todos los casos de enfermedad; tenían médico y cirujano permanente, así como los auxiliares y ayudantes necesarios; disponían de aposentos diferenciados para los enfermos contagiosos (aislados) y para los desahuciados (medida que introdujo, en 1550, el Arzobispo Fray Jerónimo de Loayza, en el Hospital de Santa Ana). Dentro de sus amplios claustros funcionaba la ropería, botica, panadería, cocinas, lavanderías generales y especiales, servicios higiénicos, redes de agua y de desagüe, campos de cultivo de plantas medicinales, crianza de aves, depósito de alimentos, etc $\left(^{8}\right)$.

Los Hospitales Mayores, por su propia condición y finalidad, estuvieron destinados a grupos humanos específicos, cuya asistencia no podía ser modificada, salvo en los casos de urgencia, hasta inicios del S. XIX, situación que contó con reglamentación especial, reconociéndose el "derecho a la asistencia inmediata".

Los Hospitales Mayores de Lima en el S. XVI fueron los siguientes: Santa Ana de los Naturales, San Andrés, Santa María de la Caridad, Espíritu Santo; en el S. XVII: San Bartolomé, para la gente de color.

\section{Hospitales menores}

Se califica así los establecimientos dedicados a tratar dolencias determinadas (lepra, en el de San Lázaro, cáncer terminal de mujeres, en Las Camilas o la Buena Muerte); se incorpora a este grupo los servicios asistenciales existentes en las Recogidas o Amparadas (para mujeres abandonadas o en estado de riesgo), en los Colegios de Doncellas y en los internados de huérfanos, expósitos, abandonados, etc. El Refugio de Incurables, después llamado de Santo Toribio de Mogrovejo, iniciado en 1669 y dedicado a crónicos, incurables y terminales, junto con las Convalecencias de indígenas y comunes, se incorpora a esta clasificación de Hospitales Menores $\left({ }^{11}\right)$. 
En forma paralela, cada Religión contaba con enfermería dentro de sus claustros, para la asistencia, cuidado y recuperación de la salud de sus miembros, novicios, legos y trabajadores, al igual que los Monasterios de religiosas de clausura, con asistencia de médicos y cirujanos contratados por periodos anuales $\left({ }^{12}\right)$.

\section{El trabajo en el hospital $\left(^{8}\right)$}

Los Hospitales Mayores de Lima y de las principales ciudades del Virreinato contaron, desde su inicio, con los servicios de médico y cirujano, quienes diariamente de mañana y tarde 'visitaban' a los enfermos de cada sala o enfermería, revisando la 'tablilla' que, a modo de información, era el resumen histórico de cada uno, colgada al pie de cada cama. La visita diaria no se realizaba aisladamente, sino con un séquito de personas formado por: enfermero mayor, enfermero ayudante, boticario y auxiliar de botica.

Cada uno de ellos cumplía una función específica: el enfermero mayor era el responsable de la sala; el ayudante realizaba las indicaciones dictadas por el médico sobre medicamentos, dieta, auxilios magnos, aplicaciones, clísteres y demás consignados en el libro diario, bajo control del enfermero mayor; el boticario era responsable de elaborar las formulaciones de uso interno o externo prescritas para cada caso, y el ayudante de botica portaba el 'cajón' con los medicamentos más el libro registro, donde se anotaba las prescripciones señaladas a cada paciente.

Era pues un equipo de personas que se movilizaba diariamente, presidido por el médico, entre las 6 y 8 de la mañana y luego de 3 a 4 de la tarde. El cirujano muchas veces formaba parte del grupo, para recibir las indicaciones del médico, dado que su labor era considerada de segundo nivel y no se equiparaba con la del médico; advertimos que las salas o enfermerías eran de enfermos en general y, la distinción de Clínica Externa e Interna, recién se introduce a partir de 1808 .
La primera separación de las enfermerías en el Hospital de Santa Ana de los naturales, fue por dolencias contagiosas (viruela, sarampión, tuberculosis, gripe, etc.) y cada una contaba con uno, dos o más enfermeros ayudantes; en tanto que, los enfermos purgados eran ubicados en aposento alejado de las salas, por razón de su propio estado. Igualmente, era completamente aislado el sector de los desahuciados, de modo que los demás enfermos no se enteraban de los decesos producidos.

Las reglas de visita del médico se encuentran reflejadas en las numerosas Constituciones de los Hospitales e informaciones de las visitas de control, que hemos revisado en nuestras investigaciones:

$1^{\circ}$ Examinar a cada uno de los pacientes, tentándoles partes de su cuerpo para mejor diagnosticar sus males; mirar las aguas menores y las albinas (heces o mayores), detenida y cuidadosamente.

$2^{\circ}$ Comunicarse con el enfermo en su propia lengua, para la mejor comprensión de sus males, de sus necesidades, preocupaciones e inquietudes.

$3^{\circ}$ Señalar el tratamiento en cada caso, ordenando los medicamentos de uso interno (jarabes, tomas, soluciones, trociscos, etc) y de aplicación externa (sinapismos, ventosas, frotaciones, ungüentos, clísteres, supositorios, emplastos, etc).

$4^{\circ}$ Hacer registrar estas indicaciones tanto en la 'tablilla' como en el libro diario por los ayudantes, en tanto que la preparación y suministro de medicinas se consignaba en el registro de la botica.

$5^{\circ}$ Señalar la dieta alimenticia a cada paciente, de acuerdo con su situación y necesidad, que debía ejecutar el enfermero mayor; que por lo general era caldo con medio pollo o bien gallina.

$6^{\circ}$ Dar indicaciones al cirujano sobre las intervenciones que debía realizar, según fuere el caso, de acuerdo con las reglas del arte quirúrgico.

$7^{\circ}$ Dedicarse con caridad y asiduidad a sus enfermos, incluso fuera de las horas de visita, tantas veces fuera necesario, además de las llamadas extraordinarias a toque especial de campana.

$8^{\circ}$ Explicar a los enfermeros ayudantes, usualmente en lengua latina, las observaciones realizadas a cada paciente y las prescripciones ordenadas; así como, recibir las informaciones proporcionadas de ellos sobre el curso de las dolencias y de los tratamientos. 
$9^{\circ}$ Estando presente el médico titular encargado de la salud y bienestar de los enfermos, no era permisible la intervención de terceros ajenos en el arte de curar ni el cambio de los tratamientos y medios curativos ordenados.

$10^{\circ} \mathrm{El}$ arte curativo del médico estaba considerado como un don o privilegio divino, como fruto de la herencia histórica grecolatina y cristiana, apoyada en los textos de Hipócrates, Galeno, Avicena, Fragoso, Montaña, Vilanova, Avila, etc. $\left({ }^{13}\right)$.

\section{La visita y la formación médica}

Cada sala o enfermería de los Hospitales Mayores podía contener entre 60 y 80 camas, situadas a ambos lados, dejando un pasillo central de circulación; las camas eran por lo general de madera, con una mesa adicional para colocar los líquidos necesarios y para las escudillas o los platos de loza en que se servía los alimentos.

El término 'covacha', utilizado entonces, corresponde a la separación o aislamiento que se hacía entre cama y cama, bien sea con tabiques de madera o de adobe, bien con cortinas corredizas, que facilitaba al médico y su equipo hacer las revisiones y exámenes de cada paciente, como la aplicación de las curaciones internas y externas.

Recordemos que los 'auxilios magnos' del arte curativo eran dos, la sangría y la purga, usadas habitualmente en forma excluyente, en función a las 'observaciones' realizadas por los ayudantes y verificadas por el médico; éste ordenaba una o varias sangrías en determinadas proporciones, generalmente del brazo opuesto al lugar de la dolencia, o bien del tobillo, examinándose la sangre extraída (densa, fibrosa, ligera, etc.) $\left({ }^{14}\right)$.

La purga era otra importante alternativa, a la que se recurría frecuentemente, mediante tomas evacuantes o purgantes, o bien por medio de enemas o lavativas a base de jabón y plantas determinadas, con el fin de despejar los humores y reducir la congestión interna, de acuerdo con la farmacopea galénica.

¿Quiénes ejecutaban los auxilios magnos? Habitualmente, eran los enfermeros ayudantes de cada sala, junto con los auxiliares y practicantes, quienes debían cumplir cuidadosamente las medidas ordenadas consignadas en el libro registro, así como las curas externas e internas en las horas y momentos correspondientes. En cambio, la sangría era realizada por el barbero o 'sangrador', mediante las lancetas o bisturíes, como se recuerda la célebre 'postemera' que utilizó San Martín de Porres, quien al morir la legó a cirujanos amigos y éstos la conservaron con gran devoción y aprecio. La misión del barbero sangrador, que se alterna con la del flebótomo, era encontrar las venas del paciente, zajarlas y extraer la sangre en las medidas ordenadas, para luego proceder al ligado, cierre y vendaje $\left({ }^{15}\right)$.

\section{Ayudantes y practicantes}

En los primeros años, éstos fueron personas del común que tenían algunos conocimientos y experiencia en los recursos del arte curativo; muchos acreditaban prácticas realizadas en hospitales hispanos, otros tan solo voluntad e intención de aprendizaje. Las informaciones producidas en todos estos casos, con pruebas instrumentales y testimoniales, contienen exhaustivos detalles dignos de ser considerados en estudios posteriores.

Al incrementarse la asistencia de los enfermos en las grandes salas o enfermerías de los Hospitales Mayores y hacerse cada vez más compleja y variada la sintomatología de las dolencias, se incorporó el 'segundo ayudante', como colaborador del enfermero mayor, que poseía un nivel más alto de formación personal, había estudiado latín y aspiraba a ser médico, por lo que ingresaba por el nivel básico de la escala. Como tal, debía realizar prácticas durante tres años como mínimo, para acceder luego a las lecciones de la Cátedra de Vísperas (generalmente por la tarde, entre 3 y $5 \mathrm{pm}$ ) y a seguido las de Prima (de mañana entre 9 y 11 am), que versaban todas ellas sobre los Tratados Hipocráticos (Aforismos, Epidemias, Legislación, Juramento, Pronóstico, Dieta, Aires, Aguas y 
Lugares, etc.) y la Doctrina de Galeno o de Arte Curativo $\left({ }^{16}\right)$.

Se conjugaba en esta forma la preparación básica general recibida por el estudiante de tres años, como mínimo, en Artes (Gramática, Latín, Filosofía), con la formación de Medicina durante tres años cuando menos, bajo dirección del Médico titular de la Cátedra, quien por lo general ejercía en uno de los Hospitales Mayores de la Ciudad, y lo destinaba a una de las plazas de 'enfermero ayudante' o como auxiliar del enfermero titular, para que durante el periodo requerido 'viera' a los enfermos, 'observara' la forma y modalidad de los tratamientos, 'conociera' el arte de curar, 'analizara' la sintomatología, los signos internos y externos, 'midiera' las reacciones a los tratamientos dispensados y 'obtuviera' conclusiones formales sustentadas en principios médicos, constituyendo en esta forma el bagaje personal de su propia experiencia, con la multiplicidad y variedad de casos presentes en el Hospital.

Vemos en esta forma que, el Médico titular y Maestro en ejercicio de su Cátedra contaba con uno o varios auxiliares, a los que dirigía y orientaba en sus prácticas diarias y compartía en lengua latina los conocimientos, las experiencias, la variada casuística producida en las enfermerías, habida cuenta del tráfico diario constante de pacientes $\left({ }^{8}\right)$.

\section{Médicos y practicantes}

Las Escuelas Prácticas de Formación y Preparación Profesional se desarrollaron en los Hospitales Mayores de Lima, como consecuencia del mayor número de estudiantes de medicina y de cirugía procedentes de todo el Virreinato del Perú, entre los siglos XVII y XVIII. Tenían necesidad de realizar su formación práctica bajo la dirección de los Médicos titulares de dichos establecimientos y completar así la exigencia contenida en las Constituciones de la Real Universidad: obtener los certificados de prácticas durante tres años como mínimo, sin cuyo requisito no podían rendir exámenes.
Para ello, se requería asimismo contar con la aquiescencia de la Hermandad de Hermanos 24 a cargo del Hospital, para su admisión y permanencia en función a los turnos horarios convenientes; sirviendo inicialmente como 'auxiliares' o 'ayudantes de enfermería', sin más beneficio que la alimentación, vestuario y cama para el descanso; aunque más adelante se les asignó una remuneración por sus servicios. La Hermandad regulaba la labor por intermedio del Diputado de semana, quien llevaba el control de todos los servidores a cargo de las enfermerías o salas y daba cuenta al Mayordomo de la buena marcha y de las incidencias.

Paralelamente, el médico titular mantenía vínculo de sujeción y mando sobre los estudiantes auxiliares o ayudantes, pues le correspondía otorgar los 'certificados de práctica' nominados por el período que estuvieron a su cargo. En otros casos, el médico del Hospital, cuando no era titular de la Cátedra, debía ante todo cumplir su misión asistencial habitual; pero, admitía a los estudiantes, reconociendo su ayuda y colaboración; en reciprocidad, les transmitía conocimientos, les recomendaba medidas y tratamientos, los hacía participar de los actos curativos y quirúrgicos, orientaba la casuística diaria, etc. En tanto que, en forma paralela, recibía sus informes y evaluaciones sobre los pacientes, con criterio más fundado que el de los simples ayudantes. Como puede advertirse, se generó una doble corriente de relación entre ambos grupos, con beneficio para la salud de los enfermos y que contribuyó a la mejor formación y preparación de los nuevos profesionales.

Gran parte de estas preceptivas surgen de los diversos expedientes seguidos sobre 'visitas' y 'estado de situación' de los Hospitales de la Ciudad de Lima, desde el S. XVI en adelante; en forma especial, fueron recogidas, analizadas y condensadas en la gran visita y reforma realizada por don Manuel Pardo y Rivadeneira, entre los años 1815 y 1817, en los cuatro Hospitales Mayores: Santa Ana, San Andrés, San Bartolomé y 
Espíritu Santo. Pardo fue un noble colaborador del Colegio de Medicina y Cirugía, pues logró que la Hermandad del Hospital de San Andrés adaptara prontamente las enfermerías de Clínica (externa e interna) y de Cirugía, a los requerimientos de don Hipólito Unanue, para la práctica diaria de los colegiales fernandinos, al expandir sus funciones el Colegio, en $1817\left({ }^{17}\right)$.

\section{¿Cuáles fueron las escuelas prácticas de medicina y cirugía?}

Hasta el momento hemos podido precisar la existencia de cinco Escuelas Prácticas de Medicina y Cirugía, que se desarrollaron en los Hospitales de Lima, desde mediados del S. XVI hasta inicios del S. XIX, por acción de los mismos Catedráticos de la Real Universidad y su vinculación con las Hermandades de Hermanos 24, cuanto por el empeño y preocupación personal de los médicos y cirujanos titulares de los establecimientos. Destaca, en todo este gran conjunto de profesionales, la figura patriarcal de D. Cosme Bueno, quien desde las enfermerías de los Hospitales de Santa Ana y de San Bartolomé, durante más de 25 años compartió su labor curativa con la docente, formativa y orientadora de los futuros profesionales, hasta su fallecimiento, en $1798\left({ }^{18}\right)$.

Reseñamos seguidamente los nombres de los más destacados representantes de estas Escuelas Prácticas en los Hospitales Mayores de la Ciudad de Lima:

\section{$1^{\text {a }}$ Hospital de Santa Ana de los Naturales}

Contaba desde su apertura, en 1549, con profesionales de primer crédito de la Ciudad, bajo control y supervigilancia del Arzobispo Fray Jerónimo de Loayza; así, se explica el ingreso, desde 1569, del médico D. Francisco Franco, graduado en la célebre Universidad de Montpellier, miembro del Claustro de la Universidad (donde se doctoró, en 1569) y Rector, en 1587. Citamos a Gaspar de Meneses, Juan de Álvarez, Francisco Gutiérrez, Alonso Gutiérrez, José Gimbert, Antonio de Albizu Juan José
Ribilla Bonet y Pueyo, el célebre grupo familiar de los Utrilla (Pedro el viejo, Pedro el mozo, Jerónimo y Miguel), Miguel de las Heras, Francisco de Vargas Machuca, José Remuzgo, hasta llegar a D. Cosme Bueno, quien se desempeñó durante más de 25 años y por sus achaques fue sustituido por su hijo Luis Bueno, el único de los nueve hijos que siguió los pasos de tan célebre padre.

Fueron destacadas las intervenciones quirúrgicas realizadas por los cirujanos pardos Utrilla, las maniobras de partos difíciles, cuanto la introducción de los más avanzados sistemas curativos por don Cosme Bueno, a partir de 1757.Gran parte de los profesionales peruanos mestizos, naturales y pardos recibió formación y preparación en el célebre local de Santa Ana ${ }^{\left({ }^{19}\right.}$ ).

\section{$2^{a}$ Hospital de San Andrés}

Desde sus comienzos, en 1550, como Hospital de hombres contó con el patrocinio del Cabildo de la Ciudad, que pagaba en forma directa al médico que asistía a los enfermos; en él se incorporó la primera Casa Enfermería establecida, en 1538, en la Rinconada de Santo Domingo. Destacamos los más importantes profesionales de la salud y miembros del Claustro de la Universidad: Hernando de Sepúlveda, Antonio Sánchez de Renedo, Álvaro de Torres, Pedro Gago de Vadillo, Melchor de Amuzgo, Juan de Vega, Francisco del Barco, José de Fontidueñas y Carrillo, Melchor Vázquez, José Miguel de Ossera y Estella, Bernabé Ortiz de Landaeta, Juan José de Aguirre, Hipólito Unanue, José Pezet, etc. $\left({ }^{20}\right)$.

Se consideró como un derecho que el médico de cámara del Virrey y del Arzobispo fuera el Catedrático de Prima de Medicina, cargo que llevaba anexo el de Protomédico General (encargado de revisar los títulos y autorizar el ejercicio profesional), junto con la titularidad del Hospital de San Andrés; medida que fue prohibida a partir de 1680 , por las implicancias que originaba y la afectación del derecho de la Hermandad. 


\section{$3^{\text {a }}$ Hospital de San Bartolomé}

Destinado a la asistencia de la gente de color, desde sus inicios en 1646, fue a no dudarlo el centro de la cirugía y de los estudios de observación médica más destacado del periodo que comentamos. La calidad y conocimientos de los médicos y cirujanos limeños, en su mayor parte, dirigidos y orientados por el sabio D. Cosme Bueno, quedó demostrada en la formación de la Escuela Médica Peruana. que Hipólito Unanue supo atraer e incorporar, desde 1808, al nuevo Colegio de Medicina y Cirugía de San Fernando $\left({ }^{17}\right)$.

Mencionamos a Pascual Sánchez, Pascual de Santa Ana, Francisco Bermejo y Roldán, Francisco de Vargas Machuca, Juan José de Villarreal, Juan de Lizeras, Pedro de Utrilla, Francisco Matute, Tomás de Obregón, Mariano Faustos, José Pastor de Larrinaga, en forma paralela con la presencia del Maestro Cosme Bueno quien, como se ha mencionado, durante un cuarto de siglo orientó la formación y preparación de los médicos y cirujanos.

Uno de ellos, el cirujano moreno José Pastor de Larrinaga, que sabía de memoria las obras de Martín Martínez, fue el gestor del Montepío de los Cirujanos del Perú, proyectó establecer un Colegio de Cirugía de San Carlos en Lima, diferenciado del de Medicina; hizo la ardorosa defensa de su gremio en la conocida "Apología de los Cirujanos del Perú» (Granada, 1791) y publicó estudios científicos en el 'Mercurio Peruano', de Lima (1791-1794).

\section{$4^{\text {a }}$ Hospital de Santa María de la Caridad}

Este establecimiento contó igualmente con una Escuela Práctica de Medicina y Cirugía; tenía el apoyo del gremio de comerciantes y del Tribunal del Consulado, en calidad de protectores del buen servicio y en especial de la atención a domicilio de las mujeres enfermas. Las principales referencias reunidas, confirman la presencia y gestión de José Ribilla Bonet y Pueyo, de los cirujanos Utrilla, José Manuel Dávalos,
Francisco Faustos, Baltasar de Villalobos, etc, quienes contribuyeron eficazmente con sus orientaciones y enseñanzas al proceso formativo de ayudantes y auxiliares, con las limitaciones y condicionamientos propios de la época; sin embargo, aquí encontramos referencias sobre la formación de las primeras 'enfermeras' o 'asistentas de las enfermas' en América Meridional, provenientes del Colegio de Doncellas de la Presentación de Na. Sa., ubicado entre la Iglesia de la Caridad y la Real Universidad $\left({ }^{21}\right)$.

\section{Hospital del Espíritu Santo de los Marinos}

La Escuela Práctica de este establecimiento rivalizó notablemente con las anteriores, desde que contaba con médicos y cirujanos parteros altamente destacados, como Felipe Bosch y Menigue, José Manuel Valdés, Félix Devotti, Juan José de Iturrizara, Pedro Echeverría, Baltasar de Villalobos y muchos más, que contribuyeron al progreso y desarrollo de las artes curativas y quirúrgicas hasta su cierre, en 1821 , y traslado, en julio de 1822, al Hospital de Bellavista, en el Callao $\left({ }^{22}\right)$.

Este Hospital llegó a tener, durante el S. XVIII, una sala especial de clínica de paga, donde se atendía casos difíciles y se realizaron audaces intervenciones quirúrgicas, conforme reseña el 'Mercurio Peruano', de Lima (1791 a 1794).

Las Escuelas Prácticas declinaron en gran medida a partir de 1793, al entrar en funciones el nuevo Anfiteatro Anatómico, edificado en el Hospital de San Andrés, y por las nuevas orientaciones científicas, disertaciones y prácticas anatómicas (autopsias) establecidas por D. Hipólito Unanue, primero como Catedrático de Anatomía y luego como Protomédico General; continuó la decadencia con el funcionamiento del Real Colegio de Medicina y Cirugía de San Fernando, a partir de 1808, eje fundamental del desarrollo integral de la Escuela Médica Peruana ( ${ }^{23}$ ).

En esta primera etapa, hasta el inicio de la República, la modalidad de asistencia y de servicio a los enfermos no se modificó 
en gran medida en los Hospitales Mayores, por haber estado a cargo de las Hermandades de Hermanos 24. Después de 1824, durante el periodo de anarquía interna, se introdujo el principio de la Beneficencia, como repercusión tardía de la Revolución Francesa, a la que se confió la administración de los servicios asistenciales, sociales y caritativos, por delegación del Estado, efectuándose la concentración de los recursos y bienes, lo que originó la extinción de las Hermandades $\left({ }^{24}\right)$.

Tuvieron que transcurrir varios años todavía, hasta 1856 , cuando se establece la Facultad de Medicina de Lima, en base al Colegio de Medicina y Cirugía, bajo el mando de Cayetano Heredia, en que se organiza los sistemas de prácticas asistenciales en los Hospitales (internos y externos) y muchos Profesores dictan sus lecciones en las salas o enfermerías a su cargo, en correspondencia con las nuevas orientaciones de la Ciencia Médica y los avances del S. XIX. Los Reglamentos Generales de Sanidad y de Hospitales dictados a fines del S. XIX establecieron las reglas de las prácticas hospitalarias y de internado, merced a convenios celebrados entre la Facultad de Medicina y la Beneficencia; importante tema técnico y social del que nos ocuparemos próximamente $\left.{ }^{24}\right)$.

En el presente estudio, se ha tratado de reseñar brevemente, el funcionamiento de las Escuelas Prácticas de Medicina y Cirugía en el Perú, en la etapa anterior a 1808, haciendo notar la valiosa contribución realizada por Maestros Médicos y Cirujanos a la formación y preparación de los nuevos profesionales, que constituyeron luego la gran Escuela Médica Peruana, dirigida por el sabio peruano D. Hipólito Unanue y Pavón.

\section{REFERENCIAS BIBLIOGRÁFICAS}

1. Eguiguren LA. Alma Mater. Lima. 1939.

2. Recopilación de las Leyes de los Reinos de Indias. Madrid.1973.

3. Guerra F. Epidemiología Americana y Filipina (1492-1898). Madrid: Ministerio de Sanidad y Consumo. 1999.
4. Guerra F. La Educación Médica en Hispanoamérica y Filipinas. Madrid: Universidad De Alcalá. 1998.

5. Guerra F. El Hospital en Iberoamérica y Filipinas. Madrid: Ministerio de Sanidad y Consumo. 1994.

6. Monardes N. La Historia Medicinal de las cosas que se traen de nuestras Indias Occidentales. Madrid: Ministerio de Sanidad y Consumo. 1989.

7. Lastres JB. Historia de la Medicina Peruana. Lima: UNMSM. 1951.

8. Rabí M. Del Hospital de Santa Ana (1549-1924) al Hospital Nacional Arzobispo Loayza (1925-1999). En: Historia de la Medicina Peruana. Tomo II. Lima: Gráfica Fina. 1999.

9. Paz Soldán CE. La Escuela Médica Peruana. Lima: Biblioteca del Centenario de Hipólito Unanue. 1932.

10. Bromley J. Lima en el S. XVI. Boletín Municipal de Lima. 1964 febrero:71-85,80-3,87-95.

11. Rabí M. El Hospital Refugio de Incurables. En: Historia de la Medicina Peruana. Tomo I. Lima. 1997.

12. Cobo B. Historia de la Fundación de Lima. Lima: Ediciones del IV Centenario. 1935.

13. Laín P. Historia Universal de la Medicina. Barcelona: Editorial Salvat. 1978.

14. Vargas F. Médicos Discursos. En: Historia de la Medicina Peruana. Tomo VI. Lima. 2005.

15. Valdizán H. Diccionario de Medicina Peruana. Lima; 1959. Tomo V y IV.

16. Eguiguren LA. Las Constituciones de la Universidad. Lima: UNMSM. 1951.

17. Rabí M. El Hospital San Bartolomé de Lima. En: Historia de la Medicina Peruana. Tomo III. Lima. 2001.

18. Paz Soldán CE. Cosme Bueno, El Precursor. Anales de la Sociedad Peruana de Historia de la Medicina. 1939;1:79-95.

19. Caviedes J. Obras completas. Biblioteca de Clásicos Peruanos. Lima: Banco Central de Reserva del Perú. 1990.

20. Rabí M. El Hospital de San Andrés de Lima, 1550 a 1875. En prensa 2006.

21. Rabí M. Investigación y estudio terminado sobre el Hospital de Santa María de la Caridad, 1559 a 1840. En prensa 2006.

22. Rabí M. Un capítulo inédito: el traslado del Hospital del Espíritu Santo de Lima a Bellavista (1750). Asclepio: Revista de la Historia de la Medicina y de la Ciencia. 1995;47:123-33.

23. Delgado G, Rabí M. Evolución histórica de la Facultad de Medicina de San Fernando, UNMSM. Lima: UNMSM. 2006.

24. Rabí M. Sociedad de Beneficencia de Lima Metropolitana. En: Historia de la Medicina Peruana. Tomo VIII. Lima. 2006.

Manuscrito recibido el 15 de febrero de 2006 y aceptado para publicación el 28 abril de 2006.

Correspondencia:

Dr. Miguel Rabi Ch.

C. Tintoretto 238, Urb. San Fco. de Borja

Lima 41, Perú

Correo-e:ecb315@suse.com.pe 